\title{
Predicción del diámetro máximo sobre muñón en árboles podados de Pinus taeda L. origen Marion en el NE de Corrientes, Argentina*
}

\author{
Prediction of the maximum diameter over stub in pruned trees of Pinus taeda L. \\ origin Marion in the NE of Comentes, Argentina. \\ HUGO E. FASSOLA ${ }^{1}$, PAULA FERRERE ${ }^{2}$, FRANCISCO RODRIGUEZ A. ${ }^{3}$ \\ ${ }^{1}$ Investigador INTA EEA Montecarlo AE N ${ }^{\circ}$ 4. (3384) Montecarlo, Misiones, Argentina. \\ Tel. / Fax 0543751480057 / 480512 E-mail: hfasola@ceel.com.ar \\ ${ }^{2}$ Becana. INTA EEA Montecarlo $\mathrm{AE} \mathrm{N}^{\circ} 4$ (3384) Montecarlo, Misiones, Argentina. \\ Tel. / Fax 0543751480057 / 480512 E-mail: ferrere@infovia.com.ar \\ ${ }^{3}$ Subgerente Papelera del Plata S.A. (3300) Posadas, Misiones, Argentina. \\ Tel.0543752450910 E-mail: frodriguez@cmpc.com.ar
}

\section{SUMMARY}

The maximum diameter over stub (dos) in trees of Pinus taeda L. origin Marion in the Northeast of the Province of Corrientes, Argentina, can be predicted with accuracy tor any pruning lift up to a maximum of four, and a dos height of $8.7 \mathrm{~m}$ above the ground. Tree variables employed for maximum dos prediction were diameter at breast height (D.B.H.), total height (Ht) and height above ground of the base of green crown before pruning (gcbbp) The best fitting and validated model against an independent database was:

$\operatorname{dos}(\mathrm{cm})=2.66941+0.374445 *$ D.B.H. $+0.50723 *$ DAPO

Where $\quad \mathrm{DAPO}=\mathrm{DBH} *\left(\frac{\mathrm{Ht}-\mathrm{gcbbp}}{\mathrm{Ht}-1,3}\right)$

D.B.H and dos are expressed in centimeters, Ht and gcbbp are in meters In the validation of the model, a mean error of $0.04 \mathrm{~cm}$ and an absolute mean error of $0.91 \mathrm{~cm}$ was obtained.

Key words: Diameter over stub, pruning, forest management, Pinus taeda L.

\section{RESUMEN}

El diámetro máximo sobre muñones (dmsm) de ejemplares de Pinus taeda L. origen Marion en el NE de Corrientes, Argentina, puede ser estimado con precisión en rodales aplicando de uno a cuatro levantes de poda, hasta una altura de $8,7 \mathrm{~m}$. Las variables de los arboles empleadas en el modelo ajustado para estimar el dmsm para un número de levantes de poda dado fueron el diámetro a la altura del pecho (dap), la altura total (H) y la altura de la base de copa verde previo a la poda (bcvpp). El mejor modelo ajustado y validado contra una muestra independiente fue: $\mathrm{dmsm}(\mathrm{cm})=2,66941+0,374445 * \mathrm{dap}+0,50723 *$ DAPO

Donde $\quad$ DAPO $=$ dap $*\left(\frac{\mathrm{H}-\mathrm{bcvpp}}{\mathrm{H}-1,3}\right)$

dmsm y dap son expresados en centímetros y tanto $\mathrm{H}$ como bcvpp en metros. Cotejada la bondad del ajuste contra una muestra independiente el error promedio fue $0,04 \mathrm{~cm}$ y el error absoluto promedio alcanzo $0,91 \mathrm{~cm}$.

Palabras claves Diámetro sobre muñón, poda, manejo forestal, Pinus taeda L

* Proyecto parcialmente financiado por SAGPyA BIRF (Convenio de Préstamo 3948 AR) Proyectos de Investigación Aplicada (PIA) en Bosques de Cultivo 20/96 


\section{INTRODUCCION}

Los rendimientos en madera libre de nudos de rollizos podados están dados por las dimensiones de los mismos, por el tamaño del "cilindro con defectos" (cd) -al que Park (1980, 1982, 1985) definió como el "cilindro que contiene la médula, los muñones de la poda, la oclusión de la herida de poda y las sinuosidades del fuste"- y por el grado de eficiencia durante la conversión (Park 1980, 1982, Turner y Pnce 1996)

Olivares P. y Meneses V (1985) definieron al diámetro sobre muñón (dsm) como el diámetro del fuste a la altura de un verticilo determinado y que ha sido podado, siendo la resultante de sumarle al diámetro del fuste el engrosamiento producido en la base de las ramas El diámetro máximo sobre muñón (dmsm) surge de la comparación de los dsm calculados para la totalidad de los verticilos podados en una oportunidad dada

Relaciones que permitan establecer las dimensiones del dmsm como resultado de determinados esquemas silvícolas en rodales podados han sido desarrolladas en forma gráfica (Sutton y Crowe 1975) y también ajusfando modelos (Knowles et al. 1987) para Pinus radiata D. Don. en Nueva Zelanda. Dichas relaciones estaban basadas en la estrecha vinculación de esta variable con el diámetro a la altura del pecho (dap), altura total $(\mathrm{H})$, diámetro de ramas en el verticilo correspondiente (dr) y la altura de poda (Hp) (Olivares y Meneses 1985).

En Argentina sólo se han establecido relaciones para algunos esquemas silvícolas de Pinus elliottii Engelm (Fassola 1992). En ejemplares podados de Pinus taeda L origen Manon se cuenta con información para el primer levante de poda en dos sitios diferentes del litoral argentino (Fassola et al. 1997, 1999b) y hasta cuatro levantes en el Departamento Iguazú, Misiones (Fassola et al. 1999c).

Considerando la relación estrecha entre el diámetro del cilindro que contiene los defectos (ded) y el diámetro máximo sobre muñones (dmsm) en Pinus taeda L. (Fassola et al. 1999a), es importante poder predecir la dimensión que alcanzará el dmsm bajo distintas alternativas silvícolas en plantaciones de Pinus taeda L. origen Marion en el NE de la provincia de Corrientes, región donde las plantaciones de esta especie se expandieron sustancialmente.
Para ello se recurrió a la información generada por un ensayo de poda y raleo ubicado en esa legión a los fines de determinar cuáles eran las variables independientes que mejor explicaban las variaciones en el tamaño del mismo y ajustar un modelo que permitiera predecir las dimensiones que alcanzaría

\section{MATERIAL Y METODOS}

La información fue recogida en un ensayo de raleo y poda en Pinus taeda L origen Marion ubicado en la localidad de Santo Tomé, provincia de Corrientes, Argentina, a $\operatorname{los} 28^{\circ} 24^{\prime}$ de lat. sur y $56^{\circ}$ de long. oeste. Este ensayo fue instalado en 1996 sobre una plantación comercial de la especie a la edad de 3 años. El último año de observación fue en 1999, correspondiendo con la aplicación del cuarto levante de poda en aquellos tratamientos donde estaba previsto. El diseño adoptado fue sistemático clinal (Alder 1980, Huxley 1983a 1983 b) considerando una faja sin raleo y fajas con un $50 \%, 75 \%$ y $87 \%$ de la densidad original. Cada faja consta de una bordura perimetral En forma perpendicular a las fajas se procedió a realizar podas de distinta intensidad y frecuencia. Los niveles de intensidad de poda se aplicaron como porcentaje de remoción de copa verde, siendo los mismos $0 \%, 30 \%, 50 \%$ y $70 \%$. Para cada intensidad de poda se ejecutaron desde dos a cuatro realces de frecuencia anual a partir del tercer año de plantado. De esta forma quedaron configuradas 5 parcelas por tratamiento y dentro de cada una de ellas se seleccionaron tres árboles a los cuales se les midieron distintas variables, totalizándose 1.260 observaciones cuyos valores mínimo y máximo se detallan en el cuadro 1.

Con esta información, de la que previamente y en forma aleatoria se extrajo una muestra independiente constituida por 130 árboles, se procedió a analizar mediante análisis de regresión cuáles eran las variables o sus transformaciones, que permitían predecir con mayor precisión el diámetro máximo sobre muñones correspondiente a cada levante de poda de los diferentes tratamientos ensayados.

De acuerdo con los antecedentes citados por Knowles et al. (1987) y Fassola et al. (1999c), se optó por ajustar modelos que incluyeran las variables dap, diámetro máximo de ramas y también 


\section{CUADRO 1}

Valores mínimos y máximos de las variables de los árboles de Pinus taeda L. origen Marion utilizadas en el modelo $(\mathrm{n}=1.260)$.

Maximum and minimum values of the variables of Pinus taeda L. origin Marion trees used in the model $(\mathrm{n}=1.260)$.

\begin{tabular}{|c|c|c|c|c|c|c|c|c|c|}
\cline { 2 - 8 } \multicolumn{1}{c|}{} & $\begin{array}{c}\text { Edad } \\
(\mathrm{años})\end{array}$ & $\begin{array}{c}\mathrm{dap} \\
(\mathrm{cm})\end{array}$ & $\begin{array}{c}\mathrm{H} \\
(\mathrm{m})\end{array}$ & $\begin{array}{c}\mathrm{Hp} \\
(\mathrm{m})\end{array}$ & $\begin{array}{c}\text { Poda } \\
(\%)\end{array}$ & $\begin{array}{c}\text { bcvpp } \\
(\mathrm{m})\end{array}$ & $\begin{array}{c}\mathrm{dmsm} \\
(\mathrm{cm})\end{array}$ & $\begin{array}{c}\text { Hdmstn } \\
(\mathrm{m})\end{array}$ & $\begin{array}{c}\mathrm{dmr} \\
(\mathrm{cm})\end{array}$ \\
\hline Máximo & 6 & 25,0 & 12,9 & 8,7 & 77,6 & 7,6 & 18,7 & 7,6 & 10,5 \\
\hline Mínimo & 3 & 3,0 & 2,5 & 1,1 & 9,1 & 0,0 & 6,4 & 0,1 & 0,4 \\
\hline
\end{tabular}

n: tamaño de la muestra; dap: diámetro a la altura del pecho, H: altura total, Hp: altura de poda, Poda: porcentaje de poda aplicado, bcvpp: altura desde el suelo de la base de copa verde previo a la poda, dmsnr diámetro máximo sobre muñones, Hdmsm: altura desde el suelo del verticilo de mayor diámetro en cada levante de poda, dmr: diámetro de la rama más gruesa, en sentido horizontal, en el verticilo podado de mayor diámetro en cada levante de poda.

una relación entre el dap y la conicidad del fuste cuya expresión original era la siguiente:

DADOS $=$ dap $*\left(\frac{H-H d m s m}{H-1,3}\right)($ Knowles et al. 1987$)$

A dicha variable se le introdujo como modificación la utilización de la altura de ocurrencia de la base de la copa verde previa a la aplicación de la poda (bcvpp), ya que mostró mejor comportamiento que la Hdmsm, denominándosela DAPO. La expresión de la variable es la siguiente:

$\mathrm{DAPO}=\operatorname{dap} *\left(\frac{\mathrm{H}-\mathrm{bcvpp}}{\mathrm{H}-1,3}\right) \quad($ Fassola et al. 1999c)

El ajuste de los modelos se efectuó combinando la variable DAPO tanto con el dap como con dmr, siendo la forma de los mismos la siguiente:

(1) $d m s m=b_{0}+b_{1} * x+b_{2} *$ DAPO

(2) $d m s m=b_{0}+b_{1} * x+b_{2} * x^{2}+b_{3} *$ DAPO

Donde :

$\mathrm{x}=$ dap $\quad \mathrm{o} \quad \mathrm{x}=\mathrm{dmr}$ y DAPO variables independientes

y

$b_{0}, b_{1}, b_{2}$ y $b_{3}$ los coeficientes de la regresión.
La bondad del ajuste de los modelos fue analizada mediante ANOVA y el empleo de la muestra independiente de 130 datos procedentes del mismo ensayo, separada en forma previa al ajuste de los modelos, que permitió determinar el error de estimación del dmsm.

La misma base de datos empleada en la construcción del modelo de predicción del dmsm fue utilizada para analizar la relación existente entre la altura de ocurrencia de éste y alguna variable del árbol, a los fines de establecer la ubicación en el fuste del dmsm en cada realce de poda.

\section{RESULTADOS Y DISCUSION}

Ajuste de un modelo de predicción del dmsm. De todos los modelos ajustados se seleccionó el (1) que incorporaba como variables independientes el dap y DAPO. En el cuadro 2 se presentan los coeficientes del mismo y en el cuadro 3 otros estadísticos que reflejan la bondad del ajuste. Aparte de haber logrado un ajuste altamente significativo, tampoco se encontró multicolinearidad entre las variables independientes, efecto que se manifestó al emplear como variable independiente dap.

La expresión final del modelo ajustado fue la siguiente:

$\mathrm{dmsm}=2,66941+0,374445 *$ dap $+0,50723 *$ DAPO

En la figura 1 se presentan los dmsm observados y los estimados mediante el empleo del mode- 


\section{CUADRO 2}

Coeficientes del modelo de predicción del dmsm en Pinus taeda L. origen Marion. Coefficients of the prediction model of maximum dos in Pinus taeda L. origin Marion.

\begin{tabular}{|l|c|c|c|c|}
\hline Parámetro & Coeficientes & ES & $\mathrm{t}$ & Valor P \\
\hline Constante & 2,669410 & 0,152 & 17,541 & 0,000 \\
dap & 0,374445 & 0,008 & 45,073 & 0,000 \\
DAPO & 0,507230 & 0,013 & 38,933 & 0,000 \\
\hline
\end{tabular}

ES: error estándar, t: valor t, P: probabilidad.

\section{CUADRO 3}

Resultados del ANOVA y estadísticos del modelo de predicción del dmsm en Pinus taeda L. origen Marion.

Results of the ANOVA and statistics of the maximum dos prediction model of Pinus taeda L. origin Marion.

\begin{tabular}{|c|c|c|c|c|}
\hline $\mathrm{R}^{2}$ & ESE & EAP & $\mathrm{F}$ & $\mathrm{P}$ \\
\hline 79,905 & 1,006 & 0,781 & 2242,71 & 0,000 \\
\hline
\end{tabular}

$\mathrm{R}^{2}$ : coef. de determinación; ESE: error estándar del estimador; EAP: error absoluto promedio; F: valor F; P: probabilidad.

lo seleccionado y en la figura 2 se puede observar la representación de los residuos, no evidenciándose una tendenciosidad manifiesta de los mismos.

Contrastando los valores estimados de dmsm por medio del modelo ajustado con los observados en la muestra independiente se constató que el error promedio del modelo fue de $0,04 \mathrm{~cm} \mathrm{y} \mathrm{el}$

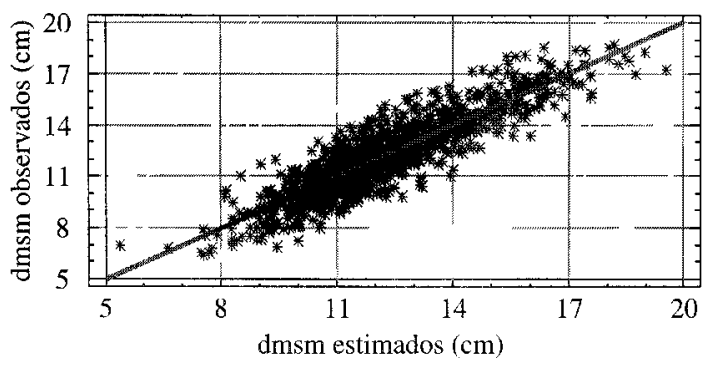

Figura 1. dmsm observados y estimados en Pinus taeda L. origen Marion.

Observed and estimated maximum dos in Pinus taeda L. origin Marion.

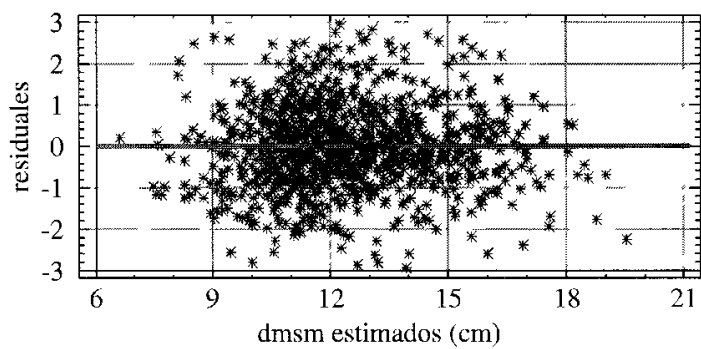

Figura 2. Residuos del modelo de predicción del dmsm en Pinus taeda L. origen Marion.

Residuals of the prediction model of maximum dos in Pinus taeda L. origin Marion.

error absoluto promedio alcanzó 0,91 cm. El $64 \%$ de los errores absolutos de estimación del dmsm de la muestra independiente fue inferior a $1 \mathrm{~cm}$ y el $82 \%$ aproximadamente, inferior a $1,5 \mathrm{~cm}$ (cuadro 4).

Analizado el error promedio de estimación del dmsm por clases diamétricas, sin considerar las clases extremas $-2,5$ y $22.5 \mathrm{~cm}$ - ya que presentaban un escaso número de observaciones -2 y 1 observación respectivamente-, se constató una muy leve sobreestimación en la clase de $7,5 \mathrm{~cm}$ y una también muy leve subestimación en las clases diamétricas de 12,5 y $17,5 \mathrm{~cm}$. Una leve tendencia al aumento de la subestimación del dmsm con la altura se pudo verificar al analizar los errores por clase de altura, aunque sin considerar las clases de 3 y 13 m por sólo contarse con 4 y 1 observación respectivamente (figura 4 ).

Determinados los errores de estimación del dmsm por clases de altura de poda se verificó una sobreestimación del mismo en la clase de hasta 1,99 $m$-con un error promedio ligeramente superior a 


\section{CUADRO 4}

Diferenciales entre dmsm observados y estimados por clases, parciales y acumulados, en Pinus taeda L. origen Marion

Differential between maximum dos observed and estimated by classes, partials and accumulates in Pinus taeda L. origin Marion.

\begin{tabular}{|c|c|c|}
\hline $\begin{array}{c}\text { Diferencial entre dmsm } \\
\text { observado y estimado }\end{array}$ & \multicolumn{2}{|c|}{$\begin{array}{c}\text { Porcentaje de error por } \\
\text { clases }(\%)\end{array}$} \\
\hline Clases (cm) & Parcial & \multicolumn{2}{|c|}{ Acumulado } \\
\hline $0,00-0,49$ & 32,8 & 32,8 \\
$0,50-0,99$ & 31,3 & 64,1 \\
$1,00-1,49$ & 17,6 & 81,7 \\
$0,50-1,99$ & 11,5 & 93,1 \\
$2,00-2,49$ & 2,3 & 95,4 \\
$2,50-2,99$ & 1,6 & 96,9 \\
$3,50-3,99$ & 1,6 & 98,5 \\
$4,00-4,49$ & 1,6 & 100,0 \\
\hline
\end{tabular}

error $(\mathrm{cm})$

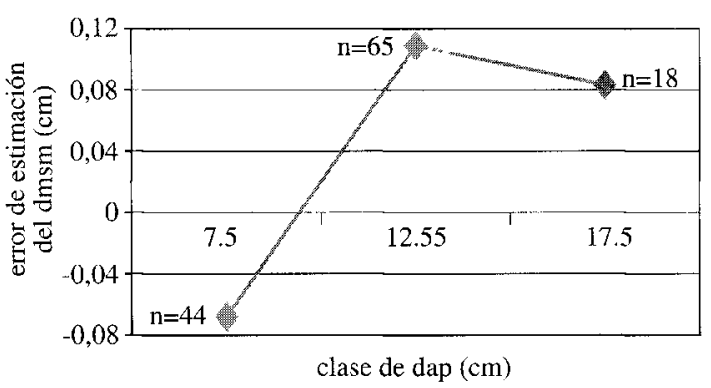

Figura 3. Error de estimación del dmsm en Pinus taeda L. origen Marion por clases de dap.

Estimation error of maximum dos in Pinus taeda L. origin Marion by DBH classes.

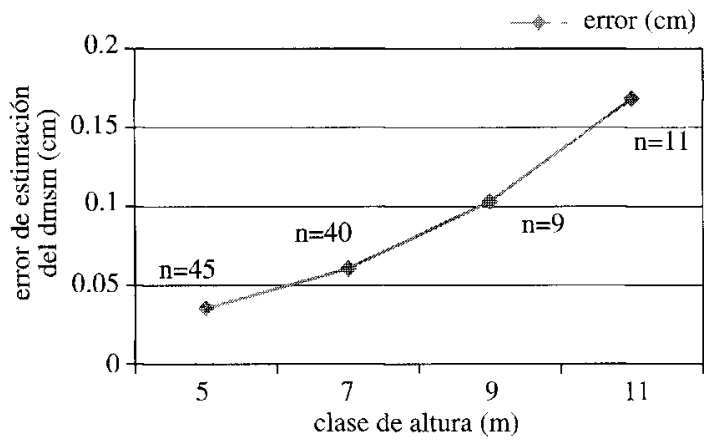

Figura 4. Error de estimación del dmsm en Pinus taeda L. origen Marion por clases de altura.

Estimation error of maximum dos in Pinus taeda L. origin Marion by height classes.
0,4 $\mathrm{cm-}$, a partir de la cual se producía una leve tendencia a la subestimación, que disminuía hacia las clases de altura mayores. Los errores correspondientes a las clases por encima de $10 \mathrm{~m} 8 \mathrm{~m}$ no fueron considerados por el escaso número de observaciones, sólo 3 (figura 5).

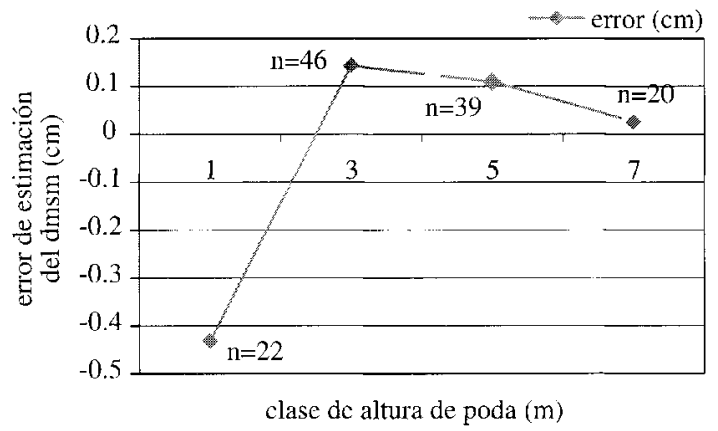

Figura 5. Error de estimación del dmsm en Pinus taeda L. origen Marion por clases de altura de poda.

Estimation error of maximum dos in Pinus taeda L. origin Marion by pruning height classes.

Altura de ocurrencia del dmsm (Hdmsm). Como resultado del análisis se determinó que para la primera poda el $\mathrm{dmsm}$ se produjo, en casi la totalidad de los casos, por debajo de $1,30 \mathrm{~m}$ aunque no se encontró asociación con ninguna variable. En el cuadro 5 se presenta la altura promedio de ocurrencia del mismo, como también de la desviación estándar y los máximos y mínimos observados.

\section{CUADRO 5}

Hdmsm en la primera poda de Pinus taeda L. origen Marion.

Height of maximum dos in the first pruning lift of Pinus taeda L. origin Marion.

\begin{tabular}{|l|c|}
\cline { 2 - 2 } \multicolumn{1}{c|}{} & Hdmsm (m) \\
\hline Promedio & 0,45 \\
Desviación estándar & 0,24 \\
Máximo & 1,38 \\
Mínimo & 0,1 \\
\hline
\end{tabular}

En las podas subsiguientes fue posible determinar que la Hdmsm se encuentra fuertemente asociada a la altura que alcanzó la poda previa. En el 
cuadro 6 se puede observar que en promedio el dmsm en las podas altas se ubicó a $14 \mathrm{~cm}$ por encima de la base de la copa verde, previo a la remoción de ramas, lo que en la práctica se consideró como el primer verticilo podado En el cuadro 7 y la figura 6 se observa la estrecha relación existente entre la altura en que se ubicó de la base de la copa activa previo a la poda (bcvpp) con la altura de ocurrencia del dmsm

\section{CUADRO 6}

Altura promedio de ocurrencia del dmsm en podas altas de Pinus taeda L origen Manon con relación a bcvpp

Mean height of maximum dos in the high pruning of Pinus taeda $\mathrm{L}$ origin Marion relative to the height of the green crown base before pruning

\begin{tabular}{|l|c|}
\hline Estadísticos & Hdmsm (m) \\
\hline Promedio & 0,14 \\
Desviación estándar & 0,23 \\
Maximo & 1,92 \\
Mínimo & 0 \\
\hline
\end{tabular}

A los mismos resultados arribaron Knowles et al. (1987) Este hecho encontraría su explicación en la concentración de auxinas de crecimiento que

\section{CUADRO 7}

Coeficientes y estadísticos de la relación $\mathrm{Hdmsm}=\mathrm{f}$ (bcvpp) en podas altas de Pinus taeda L origen Manon

Coefficients and statistics of the relationship between

Hmdos $=\mathrm{f}(\mathrm{gcbbp})$ in the high pruning of Pinus taeda $\mathrm{L}$ origin Marion.

\begin{tabular}{|l|c|}
\hline Parametro & Coeficientes \\
\hline Constante & 0,14503 \\
bcvpp & 0,992431 \\
$\mathrm{R}^{2}$ & 97,72 \\
\hline
\end{tabular}

se produciría en la base de la copa a causa de la remoción de ramas y que fuera estudiado oportunamente por Larson (1965) en ejemplares de Larix sp.

\section{CONCLUSIONES}

El diámetro máximo sobre muñones (dmsm) de ejemplares de Pinus taeda L. origen Marion podados en oportunidades sucesivas puede ser estimado, en forma empírica, para cada levante de poda con precisión mediante el empleo de un único modelo.

Tanto el dap como la variable DAPO, que establece una relación entre el dap y la conicidad del fuste, manifestaron una alta asociación con el d msm.

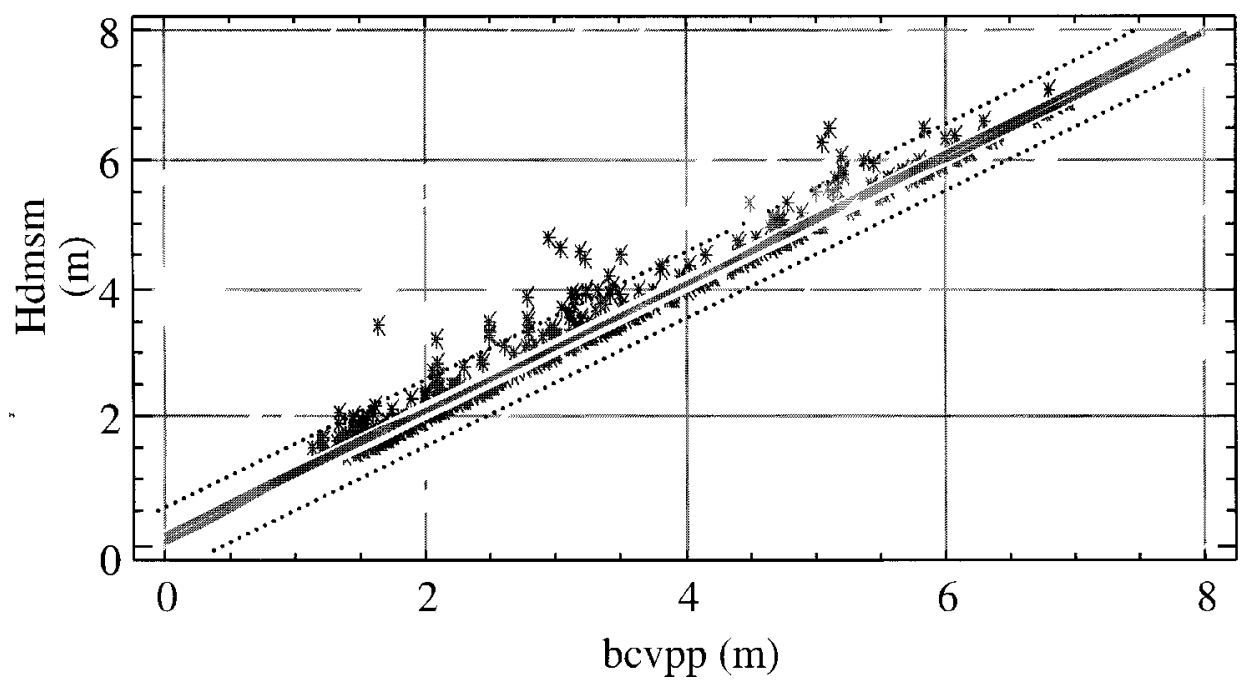

Figura 6. Valores estimados y observados de la Hdmsm en podas altas de Pinus taeda L. origen Marion con relación a la base de copa verde previa a la poda.

Estimated and observed values of the height of maximum dos in the high pruning of Pinus taeda $\mathrm{L}$ origin Marion relative to the height of the green crown base before pruning. 
Mediante el empleo de modelos, como el ajustado en este estudio, es factible programar la ejecución de esquemas de podas que mantengan el dmsm de similares dimensiones en los sucesivos levantes con el empleo de variables de fácil acceso, evitando de esa forma la presencia de cilindros que contienen los defectos agregados, a fin de maximizar el rendimiento en madera libre de nudos.

En la primera poda la altura de ocurrencia del dmsm se produjo por debajo de la altura de pecho y en los levantes sucesivos se ubicó mayormente en el primer verticilo donde comenzaba la copa activa previo a la poda.

\section{AGRADECIMIENTOS}

Se agradece la colaboración en las mediciones, aportes y sugerencias de los Ing. Ftales. D. Allegranza, A. Hernández, J. Bernio, M. Durán y E. Andenmatten.

\section{BIBLIOGRAFIA}

ALDER, D. 1980. Forest volume estimation and yield prediction. Vol. 2- Yield Prediction. Roma FAO.

HUXLEY, P. A. 1983a. Methodology for the exploration and assessment of multipurpose trees. Section Four. Part. 4E. Plant spacing. Nairobi. International Council for Research in Agroforestry.

HUXLEY, P. A. 1983b. Systematic designs for field experimentation with multipurpose trees. Section Four. Part. 4F. Designs. Nairobi. Internationa] Council for Research in Agroforestry.

FASSOLA, H. 1992. Regímenes silvícolas con producción de madera libre de nudos en Pinus elliottii Engelm en la zona central de la Prov. de Corrientes. Primer informe de avan- ce: El control del diámetro sobre muñón en $15 \mathrm{~cm}$ con tratamientos silvícolas directos. UNaM. Revista Ivyraretá 3: $28-62$.

FASSOLA, H., M. GELID DE RUI BAL. 1997. Diámetro máximo sobre muñones al momento de la primera poda en árboles dominantes de Pinus taeda L. del Depto. Iguazú, Misiones. INTA EEA Montecarlo, Informe Técnico 19: 1-7.

FASSOLA, H., J. FAHLER, P. FERRERE, D. ALLEGRANZA, J BERNIO. 1999a. Determinación del cilindro con defectos en rollizos podados de Pinus taeda L. y su relación con el rendimiento en madera libre de nudos. Proyecto de investigación aplicada 20/96. EEA INTA Montecarlo. Informe inédito $9 \mathrm{p}$.

FASSOLA, H., A. RODRIGUEZ F., D. ALLEGRANZA, P. HERNANDEZ A., P. FERRERE, M. DURAN, H. REBORATTI. 1999b. Resultados iniciales de tratamientos silvícolas directos en Pinus taeda L. origen Marion, en el NE de Corrientes. INTA EEA Montecarlo, Informe Técnico 22: $1-9$.

FASSOLA, H., P. FERRERE, M. GELID DE RUIBAL. 1999c Predicción del diámetro sobre muñones en árboles podados de Pinus taeda L. origen Marion en el Departamento Iguazú. Proyecto de investigación aplicada 20/96. EEA INTA Montecarlo. Informe inédito $11 \mathrm{p}$.

KNOWLES, R. L., G. G. WEST, A. R. KOEHLER. 1987. "Predicting diameter-over-stubs in pruned stands of radiata pine. Rotorua", Forest Research Institute Bulletin 12: 1-25.

LARSON, P. 1965. "Stem form of young Larix influenced by wind and pruning", Forest Science. Vol II 4: 412-423.

OLIVARES, P. B., M. MENESES V. 1985. Diseño de un simulador de poda. Pinus radiata Investigación en Chile. Universidad Austral de Chile. Facultad de Ciencias Forestales. Tomo II: 76-85.

PARK, J. C. 1980. "A grade index for pruned butt logs ". New Zealand Journal Forestry Science 10 (2): 419-438.

PARK, J. C. 1982. "Occlusion and the defect core in pruned radiata pine Rotorua". Forest Research Institute Bulletin 2: $1-15$.

PARK, J. C. 1985. "Recovery of clear lengths from pruned Pinus radiata saw-logs". New Zealand Journal Forestry Science 15 (2) 207-222.

SUTTON, W. R. J., J. B. CROWE. 1975. "Selective pruning of radiata pine". New Zealand Journal of Forestry Science 5 (2): 171-195

TURNER, P., C. S. PRICE. 1996. "Maximizing value of the plantation resource Part 1 - Development of a Log Grading Proposal for Pruned Softwood Logs". Suid-Afrikaanse Bosboutydskrif 176: 29-33. 